\title{
Rifampicin-resistant Mycobacterium tuberculosis among tuberculosis-presumptive cases at University of Gondar Hospital, northwest Ethiopia
}

This article was published in the following Dove Press journal:

Infection and Drug Resistance

14 June 2017

Number of times this article has been viewed

\section{Kefyalew N Jaleta ${ }^{1, *}$ \\ Mucheye Gizachew' \\ Baye Gelaw' \\ Habtie Tesfa ${ }^{2}$ \\ Alem Getaneh' \\ Belete Biadgo ${ }^{3, *}$}

'Department of Medical Microbiology, 2Department of Medical Parasitology, ${ }^{3}$ Department of Clinical Chemistry, School of Biomedical and Laboratory Sciences, College of Medicine and Health Sciences, University of Gondar, Gondar, Ethiopia

*These authors contributed equally to this work
Correspondence: Belete Biadgo

Department of Clinical Chemistry, School of Biomedical and Laboratory Sciences, College of Medicine and Health Sciences, University of Gondar, PO Box 196,

Gondar, Ethiopia

Email beletebiadigo@yahoo.com
Background: Various studies have reported that the emergence of drug-resistant tuberculosis poses a significant threat to tuberculosis-control programs worldwide. Rifampicin resistance is a surrogate marker of multidrug-resistant tuberculosis, since it reveals the presence of greater than $90 \%$ isoniazid resistance. Evidence on rifampicin-resistant Mycobacterium tuberculosis is scarce in the literature.

Objective: To determine the prevalence of rifampicin-resistant M. tuberculosis among tuberculosis-presumptive cases at the University of Gondar Hospital.

Materials and methods: A retrospective study was conducted at the University of Gondar Hospital from January 2013 to August 2015. Data were collected from registration books using a data-extraction format after securing ethical approval and checking the completeness of necessary information. Data were double-entered and rechecked to ensure accuracy and analyzed using SPSS version 20. Results were summarized using descriptive statistics. Associations were assessed using Fisher's exact test, and $P<0.05$ was considered statistically significant.

Results: A total of 1,820 M. tuberculosis-presumptive patients were included in the study. The majority of the study participants were males $(59.2 \%)$. The mean age of the participants was $36.6 \pm 15.8$ years. The preponderant age-group was $24-30$ years, with $477(23.5 \%)$ patients. The overall prevalence of $M$. tuberculosis-confirmed cases was 448 (24.6\%, 95\% CI 0.23-0.27). Of the 448 M. tuberculosis-confirmed cases, 71 (15.8\%, 95\% CI 1.12-1.19) were resistant to rifampicin. Rifampicin-resistant $M$. tuberculosis was observed among HIV seropositives (14 [18.7\%]), males (45[17.3\%]), and previously treated tuberculosis patients (61 [16.5\%]), although no significant association was found in this study.

Conclusion: The overall prevalence of $M$. tuberculosis and rifampicin resistance was found to be high in tuberculosis patients in this study. Therefore, early detection of drug-resistant M. tuberculosis should be strengthened for management of tuberculosis patients.

Keywords: University of Gondar Hospital, rifampicin-resistant, tuberculosis

\section{Introduction}

Tuberculosis (TB) is a chronic airborne infectious disease caused by the bacillus Mycobacterium tuberculosis (MTB). According to a World Health Organization (WHO) 2016 report, MTB remains a major public health problem, ranking above HIV/AIDS. It is one of the leading causes of morbidity and mortality among infectious diseases worldwide. ${ }^{1}$ The best estimate of TB deaths in 2015 was 4 million, with an additional 0.4 million deaths resulting from TB disease among HIV-positive people. In terms of cases, there were 10.4 million new TB cases, of which 5.9 million were men, 3.5 million women, and 1 million children detected. Cases that remain undetected continue 
to suffer from TB disease and also transmit the disease to their contacts. ${ }^{1,2}$

The prevention, diagnosis, and treatment of TB has become more complicated because of HIV-associated TB and multidrug resistant (MDR) TB. Many people die of TB owing to delayed diagnosis, which makes people, mainly in the sub-Saharan region, unable to reduce transmission significantly, and thus the epidemic continues. ${ }^{3}$ A global TB report estimated that there were about 230,000 (247 per 100,000 population) incident cases of TB in Ethiopia. In the same report, there were about 16,000 deaths (18 per 100,000) due to TB, excluding HIV-related deaths during the same period. ${ }^{4}$ Ethiopia ranks seventh among the world's 22 high-TB-burden countries, 10th among high-TB-pandemic countries, and fourth in sub-Saharan Africa. ${ }^{5}$

Worldwide emergence of MDR-MTB has been reported in both developed and developing countries. ${ }^{6}$ The incidence of MDR-TB is increasing, with almost half million estimated new cases in $2008 .^{7}$ Ethiopia is one of the 27 high MDRTB countries, ranked 15 th with more than 5,000 estimated MDR-TB patients each year. ${ }^{8}$

Globally, the estimated prevalence of MDR-TB was $3.3 \%$ in newly diagnosed patients in the WHO 2015 report. This was higher $(20 \%)$ in patients with a history of anti-TB treatment. ${ }^{5}$ MDR-TB is largely a consequence of poor supply management and quality of anti-TB drugs and inadequate or improper treatment. which is further worsened by HIV. Recent studies have indicated that on average, new HIV-positive TB patients are at increased risk of MDR-TB compared with HIV-negative patients. ${ }^{1,9,10}$ Poor infection-control practice has also been identified as a major contributing factor in the spread of drug-resistant TB. ${ }^{2,3}$ Based on the 2005 nationwide survey in Ethiopia, the prevalence of MDR-TB was $1.6 \%$ among new cases and $11.8 \%$ in the retreatment cases and rifampicin resistant was lower than $2 \%$ in new cases. ${ }^{11}$

A history of previous TB treatment is the strongest risk factor for progress of MDR-TB. The risk of transmission of resistant strains from close contacts is rising, because of the growing burden of MDR-TB patients ${ }^{12,13}$ and extensively DR Mycobacterium spp. ${ }^{14}$

Smear microscopy is widely used for the rapid diagnosis of TB, but it does not detect DR-MTB or sensitivity. In individuals who are coinfected with HIV, the detection rate varies between $20 \%$ and $50 \%{ }^{15}$ Results of mycobacterial culture turnaround require about 2-8 weeks, though this is not widely available in developing countries, including Ethiopia. ${ }^{15,16}$ This creates a diagnostic delay that hinders disease control, enhances transmission, and increases health-care costs. ${ }^{17}$
In Ethiopia, TB-case detection is mainly comprised of passive case finding, which is able to detect up to two-thirds of the annually estimated TB cases. TB screening among close and household contacts is one of the new approaches recommended by the national TB program to improve TBcase detection. This is usually carried out by sputum-smear microscopy and by GeneXpert MTB/RIF if the index-TB case is suspected to be a patient with DR-TB or who is at risk of harboring DR-TB. ${ }^{18}$ The national GeneXpert MTB/ RIF implementation guideline recommends its use among presumptive MDR-TB cases that include symptomatic contacts of MDR-TB cases and presumptive TB cases among HIV-positive individuals and children below 14 years of age. ${ }^{19}$

GeneXpert is an automated real-time polymerase chain-reaction assay designed for the rapid and simultaneous detection of MTB and rifampicin resistance. ${ }^{20-22}$ Most rifampin-resistant MTB-complex strains have mutations in an 81-base-pair region of the $R P O B$ gene that encodes the RNA polymerase $\beta$-subunit. This region is an ideal target for molecular tests for rifampin resistance. ${ }^{23}$ The assay amplifies a MTB-complex-specific region of the $R P O B$ gene, which is probed with molecular bonfires to detect the presence of rifampicin resistance-determining mutations. ${ }^{24}$

Continuous surveillance of the primary and acquired DR patterns of MTB is vital in assessing the efficacy of treatment regimens, as well as in detecting problems related to previous TB treatments. In Ethiopia, sputum-smear microscopy for acid-fast bacilli has been the backbone of TB-case detection in the past few decades. However, in developing countries, TB-culture and DR testing are not routinely carried out as part of the laboratory workup, owing to extreme economic disparities, low literacy, and impaired basic health-service delivery. Determining the prevalence of rifampicin-resistant MTB with advanced technology is critical to prevent drug resistance, like MDR-TB and extensively DR-TB. As far as the literature is concerned, little work has been done to document information systematically on the prevalence of rifampicin-resistant MTB using Xpert MTB/RIF in Ethiopia, particularly in Gondar. Therefore, this study aimed to determine the prevalence of rifampicin-resistant MTB among TB-presumptive cases at the University of Gondar Hospital, northwest Ethiopia.

\section{Materials and methods \\ Study design, area, and period}

A retrospective cross-sectional study was conducted from January 2013 to August 2015 at the University of Gondar Hospital TB DOTS (directly observed treatment, 
short course) Clinic laboratory. the University of Gondar Hospital is found in Gondar town. The town is located in Amhara National Regional State $727 \mathrm{~km}$ from Addis Ababa, the capital. It offers a referral service for nearly 5 million inhabitants in northwest Ethiopia. The study population were all TB-presumptive (patients with clinical signs and symptoms suggestive of TB) patients who visited the hospital during the study period. Patients presumptive for pulmonary TB and who had full documentation in the registration book were included, whereas patents who had incomplete data, eg, age, sex, GeneXpert results, were excluded from the study.

\section{Laboratory investigation}

The TB DOTS Clinic operates under the national TB- and leprosy-control program of Ethiopia, in which the diagnosis of TB is followed by Xpert MTB/RIF assay for rifampicin resistance. Samples were processed by GeneXpert MTB/ RIF assay. These were diluted and decontaminated, and the GeneXpert MTB/RIF assay was performed according to the manufacturer's manual. The Xpert MTB/RIF purifies and concentrates MTB bacilli from the samples. Genomic material is isolated from the captured bacteria by sonication and the genomic DNA subsequently amplified by polymerase chain reaction. Furthermore, the process identifies all the clinically relevant rifampicin resistance, inducing mutations in the $R P O B$ gene in the MTB genome in a real-time format using fluorescent probes called molecular beacons. Internal quality controls (sample processing control and probe check control) were used during the assay.

HIV testing was done according to the national algorithm recommended by the Federal Ministry of Health of Ethiopia. Rapid HIV tests - HIV $(1+2)$ rapid test strip and Stat-Pak were run sequentially. Positive samples were confirmed with Stat-Pak. Discordant results were resolved using a third confirmatory testing kit: HIV1/2 Uni-Gold recombinant assay. Laboratory procedures were performed according to standard operating procedures.

\section{Data collection and analysis}

Data were collected retrospectively from registration books at the University of Gondar Hospital TB DOTS Clinic using a data-extraction format after checking the completeness of the data. Seven records of study participants with incomplete data and demographic characteristics were excluded from 1,827 study participants, resulting in 1,820. Data were analyzed using SPSS version 20. Results were summarized using descriptive statistics. Association between dependent and independent variables were assessed using Fisher's exact test, and $P<0.05$ was considered statistically significant.

\section{Ethical clearance}

Data was collected after ethical clearance had been obtained from the School of Biomedical and Laboratory Sciences, College of Medicine and Health Science, University of Gondar. After discussion of the purpose and aim of the study, permission was also obtained from the head of the University of Gondar Hospital TB clinic prior to data collection. To ensure confidentiality of participants' information, anonymous typing was used, whereby the name, identification number, and any other personal identifiers were not extracted during data collection. Also, the data was kept in a confidential manner. As secondary data were used, informed consent was not sought from the study participants.

\section{Results \\ Sociodemographic and clinical characteristics}

This retrospective cross-sectional study was conducted with a total of 1,820 presumptive TB cases. Among these, $742(40.8 \%)$ were females and 1,078 (59.2\%) males. The majority of the patients, 427 (23.5\%), were in the age-group 24-30 years. Among total study participants, 315 (17.3\%) were HIV-seropositive, 622 (34.1\%) were HIV-seronegative, and the remaining $884(48.6 \%)$ had unknown HIV status (Table 1).

\section{Mycobacterium tuberculosis by sex and age}

The overall prevalence of TB was $448(24.6 \%, 95 \%$ CI $23 \%-27 \%$ ). Based on the Xpert MTB/RIF assay, the highest positive finding (29.8\%) of TB was observed in the age-group 24-30 years. The proportion of MTB was $24.1 \%$ in males and $25.3 \%$ in females. Rifampicin-resistant MTB was noted in $71(3.9 \%)$. Based on Fisher's exact test, positive test results were strongly associated with age $(P=0.001)$ (Table 2$)$.

\section{Mycobacterium tuberculosis based on HIV status and TB-treatment history}

Of the study participants, 315 (17.3\%) were HIV-positive. HIV-TB coinfection was observed in 75 (23.8\%), and 371 $(24.5 \%)$ TB cases had a history of TB treatment. However, based on Fisher's exact test, both history of treatment and HIV status of the study subjects had no statistical association with the positive findings $(P>0.05)$ (Table 2$)$. 
Table I Sociodemographic and clinical characteristics of the study participants

\begin{tabular}{llll}
\hline Variables & & $\mathbf{n}($ total I,820) & Percentage \\
\hline Sex & Male & 1,078 & 59.2 \\
Age-group, years & Female & 742 & 40.8 \\
& $<10$ & 56 & 3.1 \\
& $10-16$ & 61 & 3.4 \\
& $17-23$ & 252 & 13.8 \\
& $24-30$ & 427 & 23.5 \\
& $31-37$ & 246 & 13.5 \\
History of & $38-44$ & 238 & 13.1 \\
treatment & $45-51$ & 223 & 12.3 \\
HIV status & $52-58$ & 118 & 6.5 \\
& $>58$ & 199 & 10.9 \\
& Previously & 1,515 & 83.2 \\
& treated & & \\
Year of diagnosis & New cases & 305 & 16.8 \\
& Negative & 621 & 34.1 \\
& Positive & 315 & 17.3 \\
& Unknown & 884 & 48.6 \\
& 2013 & 225 & 12.4 \\
& 2014 & 858 & 47.1 \\
& 2015 & 737 & 40.5 \\
\hline
\end{tabular}

Table 2 Mycobacterium tuberculosis (MTB) among the study participants based on sex, age, history of TB treatment, and HIV status

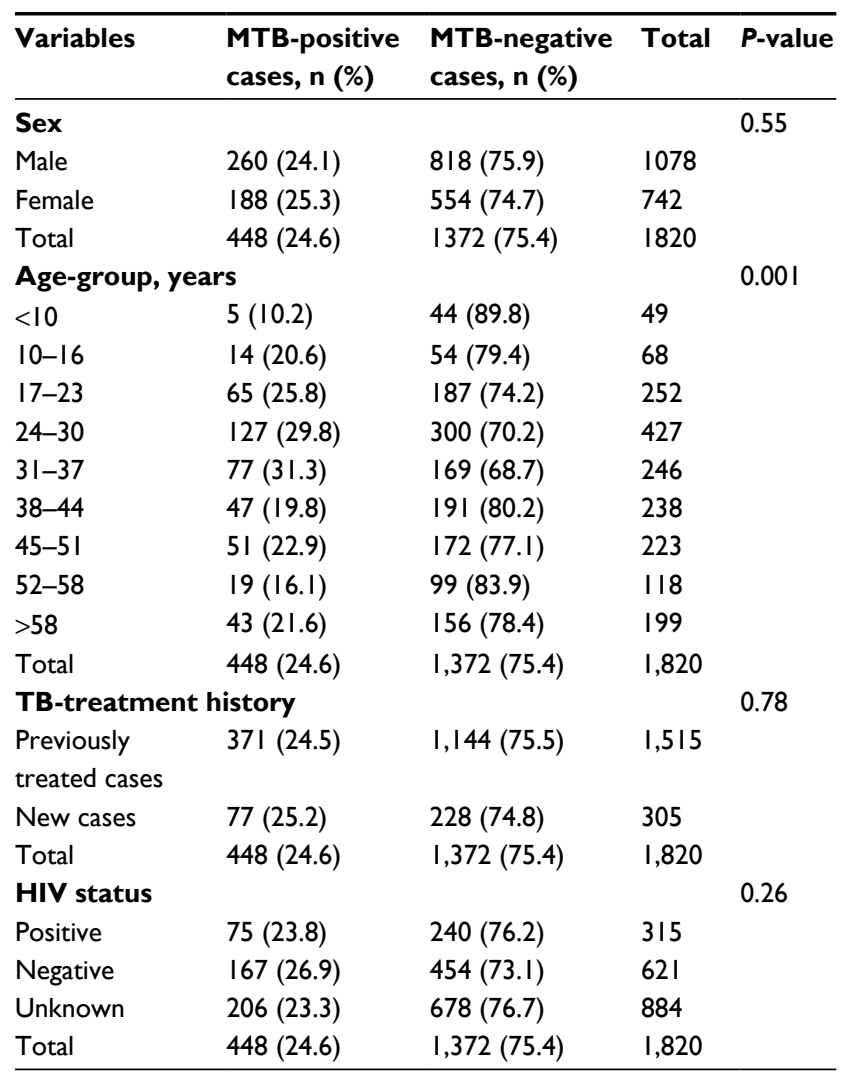

Rifampicin resistant Mycobacterium tuberculosis among TB-confirmed study participants

Of the 448 (24.6\%) TB-confirmed cases, $71(15.8 \%, 95 \%$ CI 1.12-1.19) were resistant to rifampicin. The proportion of MTB was higher 45 (17.3\%) in males, and the predominant age-groups with rifampicin-resistant TB were 17-23 and 52-58 years, 16 (24.6\%) and five (26.3\%), respectively; 75 (23.8\%) of the TB-confirmed subjects were HIV-positive. Rifampicin-resistant TB cases among HIV-positive subjects numbered 14 (18.7\%). Rifampicin-resistant MTB subjects numbered 61 (16.4\%) among previously treated TB patients (Table 3).

\section{Mycobacterium tuberculosis according to year of diagnosis}

A total of 1,820 study participants were included for Xpert MTB/RIF during 2013-2015. Of the participants enrolled,

Table 3 Rif-resistant MTB among confirmed TB patients based on sex, age, treatment history, and HIV status

\begin{tabular}{|c|c|c|c|c|}
\hline Variables & $\begin{array}{l}\text { MTB }+/ \text { Rif, } \\
\text { n (\%) }\end{array}$ & $\begin{array}{l}\text { MTB'/Rif', } \\
\text { n (\%) }\end{array}$ & $\begin{array}{l}\text { Total TB } \\
\text { positives, n (\%) }\end{array}$ & $P$-value \\
\hline Sex & & & & 0.32 \\
\hline Male & $215(82.7)$ & $45(17.3)$ & $260(58)$ & \\
\hline Female & $162(86.2)$ & $26(13.8)$ & $188(42)$ & \\
\hline Total & $377(84.2)$ & $7 I$ (I5.8) & $448(100)$ & \\
\hline \multicolumn{3}{|c|}{ Age-group, years } & & 0.06 \\
\hline$<10$ & $5(100)$ & 0 & $5(1.1)$ & \\
\hline $10-16$ & I3 (92.9) & I (7.I) & $14(3.1)$ & \\
\hline $17-23$ & $49(75.4)$ & $16(24.6)$ & $65(14.5)$ & \\
\hline $24-30$ & $103(8 I . I)$ & $24(19.9)$ & $127(28.3)$ & \\
\hline $31-37$ & $67(87)$ & $10(13)$ & 77 (I7.2) & \\
\hline $38-44$ & 45 (95.7) & $2(4.3)$ & $47(10.5)$ & \\
\hline $45-51$ & $46(90.2)$ & $5(9.8)$ & 51 (II.4) & \\
\hline $52-58$ & 14 (73.7) & $5(26.3)$ & $19(4.2)$ & \\
\hline$>58$ & $35(81.4)$ & $8(18.6)$ & $43(10)$ & \\
\hline Total & 377 (84.2) & 71 (I5.8) & $448(100)$ & \\
\hline \multicolumn{3}{|c|}{ TB-treatment history } & & 0.54 \\
\hline $\begin{array}{l}\text { Previously } \\
\text { treated cases }\end{array}$ & $310(83.6)$ & $61(16.4)$ & 371 (82.8) & \\
\hline New cases & $67(87)$ & $10(13)$ & 77 (I7.2) & \\
\hline Total & $377(84.2)$ & 71 (I5.8) & $448(100)$ & \\
\hline HIV status & & & & 0.33 \\
\hline Positive & 61 (8I.3) & 14 (I8.7) & $75(16.7)$ & \\
\hline Negative & I 46 (87.4) & $21(12.6)$ & $167(37.3)$ & \\
\hline Unknown & $170(82.5)$ & $36(17.5)$ & $206(46)$ & \\
\hline Total & $377(84.2)$ & $7 \mid$ (I5.8) & $448(100)$ & \\
\hline
\end{tabular}




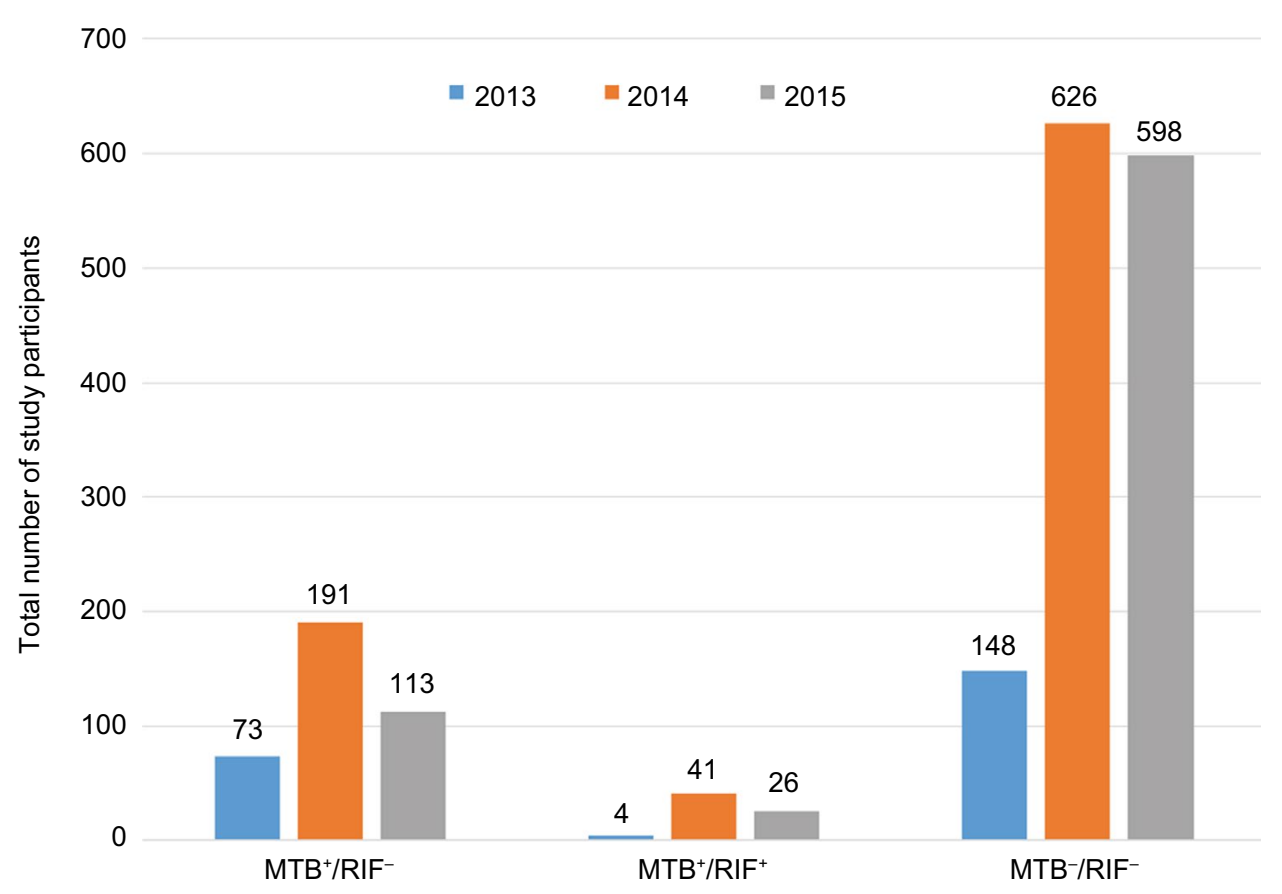

Figure I Rif-resistant MTB based on year of diagnosis.

Notes: MTB+/RIF-, MTB-positive, but not Rif-resistant. MTB'/RIF+: MTB-positive and Rif-resistant.

Abbreviations: MTB, Mycobacterium tuberculosis; Rif, rifampicin.

$448(24.6 \%)$ were positive for TB on GeneXpert, of which $71(3.9 \%)$ were rifampicin-resistant. The trend in prevalence of TB was relatively higher in 2014 (Figure 1).

\section{Discussion}

The impact and management of rifampicin-resistant TB have not been deeply studied compared to MDR-TB, which is well known to be associated with poor TB-treatment outcome. This study reveals an overall prevalence of TB among presumptive cases of 448 (24.6\%). This finding is higher than the previous studies conducted in southern Ethiopia $(16.5 \%, 19.4 \%){ }^{25-27}$ However, our finding is lower than a study conducted in East Gojjam, Ethiopia of 124 (32.2\%). ${ }^{28}$ A probable reason for this variation might have been that we included presumptive cases to identify MTB, while other studies included identified cases of TB to check GeneXpert differences in study population. The method of diagnosis and study setting may account for the variation in prevalence across studies.

According to our study, the disease predominated slightly among females: 188 (25.3\%). This is comparable with a study conducted in southern Ethiopia, ${ }^{25}$ contrary reports from the WHO, ${ }^{1,4,5}$ and a population-based prevalence survey of TB in the Tigray region of Ethiopia, which confirmed TB was higher among males (352 of 100,000). ${ }^{29}$ This difference in sex incidence might be due to health-seeking behavior, environmental factors, and the higher exposure of males to different factors that pose a risk of acquiring the TB bacilli. Even if it is difficult to compare with previous reports directly, as different studies used different cutoff points for age-groups, based on our study result, TB was more prevalent among the productive age group of study participants (127 [29.8\%]) and those with rifampicin resistance (16 [24.6\%]). This is in agreement with studies conducted in Yirgalem Hospital in southern Ethiopia and the Agaro Teaching Health Center in southwestern Ethiopia. ${ }^{25,27}$ This might be due to more exposure to the outer environment, high workload, and wide range of mobility in younger age-groups.

We found higher levels of resistance to rifampicin, 71 (3.9\%), among the participants, and 71 (15.8\%) among TB confirmed cases, almost equivalent (3.4\%) to the study conducted in Yirgalem, southern Ethiopia ${ }^{25}$ and higher than other studies conducted in Ethiopia, where resistance was 2.5\% in the northern, $1.7 \%$ in the east, $1.9 \%$ (primary resistance) in the northwest Ethiopia, and 2.59\% in East Gojjam. ${ }^{25,28-34}$ This variation might be due to disparities in awareness of studied populations about drug resistance, access to healthcare facilities, disorganized patient diagnosis, treatment, and follow-up, and poor patient adherence, which may have contributed to the higher prevalence of rifampicin-resistant TB in our study. The higher level of rifampicin resistance might be due to the fact that rifampicin is currently used for 
the treatment of many other infectious diseases in this study area. Since rifampicin is the most vital drug to treat TB, the advent of resistance to this drug has enormous implications for TB-control programs.

Prevalence in this study was higher than studies conducted in South Africa during 2007-2009 with an overall proportion of rifampicin resistance $8.8 \%$, studies in Kenya of $6.5 \%$, India of $4.69 \%$ ), and Iran of $7.4 \%$, with an average rate of $5 \%-10 \% .{ }^{35-38}$ On the other hand, our finding was lower than a study conducted in Nigeria $(18.8 \%),{ }^{39}$ but comparable to a study conducted in Sudan (15.5\%). ${ }^{40}$ Probable reasons for this would be differences in patient selection, sample size (small samples could overrepresent the proportion), irregular supply of anti-TB drugs, poor TB case management (inadequate diagnosis, treatment, and follow-up), and poor treatment compliance. Also, rifampicin has several adverse effects that could result in patient nonadherence, and hence may lead to an increase in resistant strains.

Studies conducted in Iran and Nigeria revealed that none of the isolates was resistant to rifampicin alone. ${ }^{41,42}$ This might be due to a regular supply of anti-TB drugs and adequate diagnosis, treatment, and follow-up of TB patients. In the current study, males were more likely to have rifampicin resistance (45 [17.3\%]). TB rates in South Africa have shown higher prevalence in males than in females. ${ }^{35}$ This sex-incidence variation might be associated with high healthseeking behavior in males. ${ }^{43}$

Studies around the world have shown significant variations in the prevalence of resistance to anti-TB drugs in patients previously treated for TB. Based on a 2005 nationwide survey, Ethiopia is also among the high-TB/HIV-burden countries, with a TB-HIV coinfection rate over $10 \%$, with MDR-TB prevalence $1.6 \%$ and $11.8 \%$ among new and retreatment cases, respectively, and rifampicin resistance lower than $2 \%$ in new cases. ${ }^{11}$ With respect to treatment history, the isolates showed different resistance patterns for rifampicin $61(16.5 \%)$ for previously treated cases, and the remainder were new cases (ten [13\%]) from TB-confirmed study participants.

Our study showed that there were high levels of drug resistance among those previously treated for TB. This finding is higher compared to other studies in Kenya, with prevalence of resistance of $9.9 \%$ and 0 for retreatment and new cases, respectively, and Bujumbura, Burundi, with 2\% from new cases and 15\% from previously treated cases, ${ }^{44,45}$ East Gojjam (2.59\% among new cases, 0 among retreatment cases), ${ }^{28}$ and western India (retreated cases $9 \%$ rifampicinresistant). ${ }^{46,47}$ Other studies have reported an association between previous history of TB treatment and anti-TB-drug resistance. ${ }^{48,49}$ In addition, a study in Palermo, Italy reported a wide heterogeneity among the MTB strains observed, which illustrates rapid changes in TB epidemiology. ${ }^{50}$ This indicates the existence of ongoing transmission of DR strains, and could indicate weakness in TB prevention and control measures, as it is convoyed by resistance to rifampicin in new cases as well. This indicates that transmission of MDR-TB has a considerable role in this epidemic, and historical high frequency of treatment abandonment may contribute toward high levels of resistance.

In our study, the overall prevalence of TB was high, and the prevalence of HIV-TB coinfection was also high $(23.8 \%)$. Rifampicin resistance in HIV-TB coinfected participants was $18.7 \%$, which is higher than a 2005 estimate of nationwide rifampicin resistance under $2 \%$ in new cases. ${ }^{11} \mathrm{~A}$ high prevalence of rifampicin resistance among isolates from TB-HIV coinfected patients has been also reported from Brazil. ${ }^{51}$ Resistance to specific anti-TB drugs among HIV-infected patients was evident in studies on global resistance rate to rifampicin alone $\left(0.2 \%-9.1 \%{ }^{51}\right.$ A survey of TB isolates collected in the US between 1993 and 1996 documented rifampicin monoresistance of $2.6 \%$ in HIV-positive cases and only $0.2 \%$ in HIV-negative cases. ${ }^{52}$ This might be due to HIV infection, which may lead to malabsorption of anti-TB drugs, especially rifampicin, with adverse effects leading patients to nonadherence and subsequent drug resistance and treatment failure. This study tried to assess a heretofore untouched area. This is very valuable and instructive, and could aid in comprehension for health professionals and policy makers to address the problem. Data incompleteness and poor document-retention systems were a limitation of this study, since it involved secondary data.

\section{Conclusion}

The prevalence of MTB and rifampicin resistance were high in this study. Therefore, early detection of DR-MTB should be strengthened for management of TB patients. HIVcoinfected and previously treated patients were more likely to develop rifampicin resistance. Adequate treatment periods and observation of TB cases with strict implementation of directly observed treatments should be considered, and the HIV status of all patients and duration of treatment needs to be documented.

\section{Acknowledgments}

We would like to thank the research and ethics committee of the School of Biomedical and Laboratory Science, College 
of Medicine and Health Sciences, University of Gondar, for giving us this opportunity. Last but not least, we would like to acknowledge University of Gondar Hospital TB clinic laboratory staff members for their cooperation during data collection.

\section{Author contributions}

$\mathrm{KNJ}$ and $\mathrm{BB}$ conceived and designed the study, performed analysis, interpreted data, and drafted the manuscript. MG, BG, HT, and AG assisted with the design, performed analysis and interpretation of data, and critically reviewed the manuscript. KNJ and BB are joint first authors of this article. All authors read and approved the submitted version of the manuscript.

\section{Disclosure}

The authors report no conflicts of interest in this work.

\section{References}

1. World Health Organization. Global Tuberculosis Report 2016. Geneva: WHO; 2016.

2. Morrison J, Pai M, Hopewell PC. Tuberculosis and latent tuberculosis infection in close contacts of people with pulmonary tuberculosis in low-income and middle-income countries: a systematic review and meta-analysis. Lancet Infect Dis. 2008;8:359-368.

3. Piatek AS, van Cleeff M, Alexander H, et al. GeneXpert for TB diagnosis: planned and purposeful implementation. Glob Health Sci Pract. 2013;1:18-23.

4. World Health Organization. Definitions and reporting framework for tuberculosis - 2013 revision. 2013. Available from: http://www.who. int/tb/advisory_bodies/impact_measurement_taskforce/meetings/ accra2013_9_revised_who_definitions_falzon.pdf?ua $=1$. Accessed May 23, 2017.

5. World Health Organization. Global Tuberculosis Report 2015. Geneva: WHO; 2015.

6. Sethi S, Sharma S, Sharma SK, Meharwal SK, Jindal SK, Sharma M. Drug susceptibility of Mycobacterium tuberculosis to primary ant tubercular drugs by nitrate reductase assay. Indian $J$ Med Res. 2004;120:468-471.

7. World Health Organization. Multidrug and Extensively Drug-Resistant TB (M/XDR-TB): Global Report of Surveillance and Response. Geneva: WHO; 2009.

8. Ethiopia Federal Ministry of Health. Guideline for Program and Clinical Management of Drug-Resistant Tuberculosis. 5th ed. Addis Ababa: FMOH; 2009.

9. Mesfin YM, Hailemariam D, Biadglign S, Kibret KT. Association between HIV/AIDS and multi-drug resistance tuberculosis: a systematic review and meta-analysis. PLoS One. 2014;9:e82235.

10. van den Hof S, Tursynbayeva A, Abildaev T, Adenov M, Pak S, Ismailov S. HIV and multidrug-resistant tuberculosis: overlapping risk factors. Eur Respir J. 2015;45:567-569.

11. Ethiopia Federal Ministry of Health. National Guideline on Programmatic Management of Drug-Resistant Tuberculosis in Ethiopia. 2nd ed. Addis Ababa: FMOH; 2013.

12. Gandhi NR, Nunn P, Dheda K, et al. Multidrug-resistant and extensively drug-resistant tuberculosis: a threat to global control of tuberculosis. Lancet. 2010;375:1830-1843.

13. Sharma KS, Kaushik G, Jha B, et al. Prevalence of multidrug-resistant tuberculosis among newly diagnosed cases of sputum-positive pulmonary tuberculosis. Indian J Med Res. 2011;133:308-311.
14. Andrews RJ, Shah NS, Gandhi N, Moll T, Friedland G. Multidrugresistant and extensively drug-resistant tuberculosis: implications for the HIV epidemic and antiretroviral therapy rollout in South Africa. J Infect Dis. 2007;96 Suppl 3:S482-S490.

15. Getahun H, Harrington M, O’Brien R, Nunn P. Diagnosis of smearnegative pulmonary tuberculosis in people with HIV infection or AIDS in resource-constrained settings: informing urgent policy changes. Lancet. 2007;369:2042-2049.

16. Pai M, Kalantri S, Dheda K. New tools and emerging technologies for the diagnosis of tuberculosis - part II: active tuberculosis and drug resistance. Expert Rev Mol Diagn.2006;6:423-432.

17. World Health Organization. Pathways to Better Diagnostics for Tuberculosis: A Blueprint for the Development of TB Diagnostics. Geneva: WHO; 2009.

18. Ethiopia Federal Ministry of Health. Guidelines for Clinical and Programmatic Management of TB, TB/HIV, and Leprosy in Ethiopia. Addis Ababa: FMOH; 2016.

19. Ethiopia Federal Ministry of Health. Implementation Guideline for GeneXpert MT B/RIF Assay in Ethiopia. Addis Ababa: FMOH; 2014.

20. Helb D, Jones M, Story E, et al. Rapid detection of Mycobacterium tuberculosis and rifampin resistance by use of on-demand, near-patient technology. J Clin Microbiol. 2010;48:229-237.

21. Boehme CC, Nabeta P, Hillemann D, et al. Rapid molecular detection of tuberculosis and rifampin resistance. $N$ Engl $J$ Med. 2010;363:1005-1015.

22. Van Rie A, Page-Shipp L, Scott L, Sanne I, Stevens W. Xpert MTB/RIF for point of care diagnosis of TB in high-HIV burden, resource limited countries: hype or hope? Expert Rev Mol Diagn. 2010;10:937-946.

23. Sam IC, Drobniewski F, More P, Kemp M, Brown T. Mycobacterium tuberculosis and rifampin resistance, United Kingdom. Emerg Infect Dis. 2006;12:752-759.

24. El-Hajj HH, Marras SA, Tyagi S, Kramer FR, Alland D. Detection of rifampin resistance in Mycobacterium tuberculosis in a single tube with molecular beacons. J Clin Microbiol. 2001;39:4131-4137.

25. Hordofa MW, Adela TB. Prevalence of refampcin [sic] mono resistant Mycobacterium tuberculosis among suspected cases attending at Yirgalem Hospital. Clin Med Res. 2015;4:75-78.

26. Zerihun Z, Girmay M, Adane W, Gobena A. Prevalence of pulmonary tuberculosis and associated risk factors in prisons of Gamo Goffa zone, south Ethiopia: a cross- sectional study. Am J Health Res. 2014;2:291-297.

27. Ali H, Zeynudin A, Mekonnen A, Solomon A, Ali S. Smear positive pulmonary tuberculosis prevalence amongst patients at Agaro Teaching Health Center, south west Ethiopia. Ethiop J Health Sci. 2012;22: 71-76.

28. Adane K, Ameni G, Bekele S, Abebe M, Asefa A. Prevalence and drug resistance profile of Mycobacterium tuberculosis isolated from pulmonary tuberculosis patients attending two public hospitals in East Gojjam zone, northwest Ethiopia. BMC Public Health. 2015; 15:572.

29. Berhe G, Enqueselassie F, Hailu E, et al. Population-based prevalence survey of tuberculosis in the Tigray region of Ethiopia. BMC Infect Dis. 2013; $13: 448$.

30. Nigus DM, Lingerew WM, Beyene BA, et al. Prevalence of multi drug resistant tuberculosis among presumptive multi drug resistant tuberculosis cases in Amhara National Regional State, Ethiopia. J Mycobact Dis. 2014;4:1000152.

31. Seyoum B, Demissie M, Worku A, Bekele S, Aseffa A. Prevalence and drug resistance patterns of Mycobacterium tuberculosis among new smear positive pulmonary tuberculosis patients in eastern Ethiopia. Tuberc Res Treat. 2014;2014:753492.

32. Tessema B, Beer J, Emmrich F, et al. First- and second-line antituberculosis drug resistance in northwest Ethiopia. Int J Tuberc Lung Dis. 2012;16:805-811.

33. Bruchfeld J, Aderaye G, Palme IB, et al. Molecular epidemiology and drug resistance of Mycobacterium tuberculosis isolates from Ethiopian pulmonary tuberculosis patients with and without human immunodeficiency virus infection. J Clin Microbiol. 2002;40:1636-1643. 
34. Yimer SA, Agonafir M, Derese Y, Sani Y, Bjune GA, Holm-Hansen C. Primary drug resistance to anti-tuberculosis drugs in major towns of Amhara region, Ethiopia. APMIS. 2012;120:503-509.

35. Coovadia YM, Mahomed S, Pillay M, Werner L, Mlisana K. Rifampicin mono-resistance in Mycobacterium tuberculosis in KwaZulu-Natal, South Africa: a significant phenomenon in a high prevalence TB-HIV region. PLoS One. 2013;8:e77712.

36. Ndung'u PW, Kariuki S, Ng'ang'a Z, Revathi G. Resistance patterns of Mycobacterium tuberculosis isolates from pulmonary tuberculosis patients in Nairobi. J Infect Dev Ctries. 2012;6:33-39.

37. Lahiri S, Mukherjee A, Hazra S, Jana P, Roy S, Saha BK. First-line anti-tubercular drug resistance of mycobacterial strains from re-treatment cases that were smear-positive at 4th month onwards under the Revised National Tuberculosis Control Program. Lung India. 2015;32: 127-131.

38. Velayati AA, Farnia P, Mozafari M, et al. High prevalence of rifampinmonoresistant tuberculosis: a retrospective analysis among Iranian pulmonary tuberculosis patients. Am J Trop Med Hyg. 2014;90:99-105.

39. Lawn SD, Brooks SV, Kranzer K, et al. Screening for HIV Associated Tuberculosis and rifampicin resistance before antiretroviral therapy using the Xpert MTB/RIF assay: a prospective study. PLoS Med. 2011;8:e1001067.

40. Nour EM, Saeed EM, Zaki AZ, Saeed NS. Drug resistance patterns of Mycobacterium tuberculosis isolates from patients with pulmonary tuberculosis in the Sudan. IOSR J Dent Med Sci. 2015;14:17-19.

41. Namaei MH, Sadeghian A, Naderinasab M, Ziaee M. Prevalence of primary drug resistant Mycobacterium tuberculosis in Mashhad, Iran. Indian J Med Res. 2006;124:77-80.

42. Otu A, Umoh V, Habib A, Ameh S, Lawson L, Ansa V. Drug resistance among pulmonary tuberculosis patients in Calabar, Nigeria. Pulm Med. 2013;2013:235190.
43. Austin J, Dick J, Zwarenstein M. Gender disparity amongst TB suspects and new TB patients according to data recorded at the South African Institute of Medical Research laboratory for the Western Cape Region of South Africa. Int J Tuberc Lung Dis. 2004;8:435-439.

44. Ng'ang'a ZW, Nyang'au LO, Amukoye E. Determining first line antituberculosis drug resistance among new and re-treatment tuberculosis/ human immunodeficiency virus infected patients, Nairobi Kenya. Int J Sci Basic Appl Res. 2015;19:426-437.

45. Sanders M, Van Deun A, Ntakirutimana D, et al. Rifampicin monoresistant Mycobacterium tuberculosis in Bujumbura, Burundi: results of a drug resistance survey. Int J Tuberc Lung Dis. 2006;10:178-183.

46. Sethi S, Mewara A, Dhatwalia SK, et al. Prevalence of multidrug resistance in Mycobacterium tuberculosis isolates from HIV seropositive and seronegative patients with pulmonary tuberculosis in north India. BMC Infect Dis. 2013;13:137.

47. Pradhan N, Desai S, Kagal A, et al. Patterns of TB drug-resistance in a tertiary care facility in Pune, India. Clin Microbiol. 2013;2:1000123.

48. Demissie M, Gebeyehu M, BerhaneY. Primary resistance to anti-tuberculosis drugs in Addis Ababa, Ethiopia. Int JTuberc Lung Dis. 1997;1:64-67.

49. Abate G, Miörner H. Susceptibility of multidrug-resistant strains of Mycobacterium tuberculosis to amoxicillin in combination with clavulanic acid and ethambutol. JAntimicrob Chemother. 1998;42:735-740.

50. Bonura C, Gomgnimbou MK, Refrégier G, et al. Molecular epidemiology of tuberculosis in Sicily, Italy: what has changed after a decade? BMC Infect Dis. 2014;14:602.

51. Bammann RH, Zamarioli LA, Pinto VS, et al. High prevalence of drug-resistant tuberculosis and other mycobacteria among HIV-infected patients in Brazil: a systematic review. Mem Inst Oswaldo Cruz. 2010;105:838-841.

52. Moore M, Onorato IM, McCray E, Castro KG. Trends in drug-resistant tuberculosis in the United States, 1993-1996. JAMA. 1997;278:833-837.
Infection and Drug Resistance

\section{Publish your work in this journal}

Infection and Drug Resistance is an international, peer-reviewed openaccess journal that focuses on the optimal treatment of infection (bacterial, fungal and viral) and the development and institution of preventive strategies to minimize the development and spread of resistance. The journal is specifically concerned with the epidemiology of antibiotic

\section{Dovepress}

resistance and the mechanisms of resistance development and diffusion in both hospitals and the community. The manuscript management system is completely online and includes a very quick and fair peerreview system, which is all easy to use. Visit http://www.dovepress.com/ testimonials.php to read real quotes from published authors. 